\title{
Effective Dose in Nuclear Medicine Studies and SPECT/CT: Dosimetry Survey Across Quebec Province
}

\author{
Mathieu Charest ${ }^{1}$ and Chantal Asselin ${ }^{2}$ \\ ${ }^{I}$ Nuclear Medicine Service, Hôpital du Sacré-Cour de Montréal, Montreal, Quebec, Canada; and ${ }^{2}$ Collège Ahuntsic, Montreal, \\ Quebec, Canada
}

The aims of the current study were to draw a portrait of the delivered dose in selected nuclear medicine studies in Québec province and to assess the degree of change between an earlier survey performed in 2010 and a later survey performed in 2014. Methods: Each surveyed nuclear medicine department had to complete 2 forms: the first, about the administered activity in selected nuclear medicine studies, and the second, about the CT parameters used in SPECT/CT imaging, if available. The administered activities were converted into effective doses using the most recent conversion factors. Diagnostic reference levels were computed for each imaging procedure to obtain a benchmark for comparison. Results: The distributions of administered activity in various nuclear medicine studies, along with the corresponding distribution of the effective doses, were determined. Excluding ${ }^{131} \mathrm{I}$ for thyroid studies, ${ }^{67} \mathrm{Ga}$-citrate for infectious workups, and combined stress and rest myocardial perfusion studies, the remainder of the $99 \mathrm{mTc}$-based studies delivered average effective doses clustered below $10 \mathrm{mSv}$. Between the 2010 survey and the 2014 survey, there was a statistically significant decrease in delivered dose from 18.3 to $14.5 \mathrm{mSv} .{ }^{67} \mathrm{Ga}$-citrate studies for infectious workups also showed a significant decrease in delivered dose from 31.0 to $26.2 \mathrm{mSv}$. The standardized CT portion of SPECT/CT studies yielded a mean effective dose 14 times lower than the radiopharmaceutical portion of the study. Conclusion: Between 2010 and 2014, there was a significant decrease in the delivered effective dose in myocardial perfusion and ${ }^{67} \mathrm{Ga}$-citrate studies. The CT portions of the surveyed SPECT/CT studies contributed a relatively small fraction of the total delivered effective dose.

Key Words: dosimetry; SPECT; SPECT/CT; conversion factor; effective dose; activity

J Nucl Med Technol 2018; 46:107-113

DOI: 10.2967/jnmt.117.202879

I n 2010, we performed an initial survey in Québec province about administered doses in nuclear medicine. The doses delivered were found to be quite variable among centers. After that time, newer imaging technology became available

\footnotetext{
Received Sep. 26, 2017; revision accepted Nov. 28, 2017.

For correspondence or reprints contact: Mathieu Charest, Hôpital du SacréCœur de Montréal, 5400 Boul. Gouin Ouest, Montreal, Quebec, Canada, H4J1C5.

E-mail: mathieu.charest@gmail.com

Published online Dec. 22, 2017.

COPYRIGHT (c) 2018 by the Society of Nuclear Medicine and Molecular Imaging.
}

with the advance of resolution recovery software, semiconductor detectors (Discovery NM530c; GE Healthcare), optimization software, and collimators (IQ SPECT; Siemens). In addition, camera providers and the medical community became more aware of the importance of dosimetry and protocol optimization. The results of our initial survey were described in educational presentations at provincial and national scientific meetings. In 2014, we decided it was time for a followup survey to assess the result of this educational process.

The aims of the 2014 survey were, first, to draw a portrait of the delivered dose to patients in nuclear medicine in 2014 across the province and, second, to assess the degree of changes between the 2010 and 2014 surveys.

\section{MATERIALS AND METHODS}

Between May and June 2014, we sent a survey to nuclear medicine departments across the province. Each center had to complete 2 standardized forms: the first, about the administered activity in selected diagnostic nuclear medicine studies, and the second, about the CT parameters used in SPECT hybrid cameras with CT imaging (SPECT/CT), if such technology was available at the center. For each nuclear medicine procedure, the responders first had to indicate the most commonly used radiotracer and the administered activity. They also had to specify the imaging device, such as a standard scintillation camera, semiconductor camera, or PET camera. In addition, they had to specify whether count recovery software or a special collimator was used. For selected SPECT/CT studies of the head and neck, thorax, abdomen, or extremities, they had to indicate the brand of the camera, the current, the modulation of the current, the tension, as well as the pitch, rotation time, slice thickness, and collimation. They also had to indicate the usual length of a scan and the typical CT dose index (CTDI) and dose-length product (DLP) for each of the protocols in question.

The institutional review board approved this retrospective study, and the requirement to obtain informed consent was waived. The statistical analyses that were performed included subgroup and group averaging, SD calculation, and percentile determination. To convert administered activity ( $\mathrm{mCi}$ or $\mathrm{MBq}$ ) into effective dose $(\mathrm{mSv})$, the conversion factors in publication 128 of the International Commission on Radiological Protection (ICRP) (l) were used. When a particular conversion factor for a given radiopharmaceutical was not available in that publication, an earlier publication-ICRP 53,80 , or $106(2-4)$-was used instead.

To compare the effective dose received for various CT settings in SPECT/CT imaging, we calculated the average DLP for each procedure. This average was determined from the average CDTIs, 
which were based on the survey responses about acquisition parameters, and multiplied by a standard $20-\mathrm{cm}$ scanning length. We decided to use a standard scanning length to remove this variable from the equation when comparing the effects of the other CT parameters among them. DLPs $(\mathrm{mGy} \times \mathrm{cm})$ were multiplied by the appropriate conversion factors to obtain effective dose $(\mathrm{mSv})$. The conversion factors were retrieved from the Imaging Performance Assessment of CT scanners (ImPACT) CT dosimetry tool, version 1.0.3 (5), using the National Radiological Protection Board SR250 dose distribution data obtained from Monte Carlo calculation.

To compare the effective dose delivered for each nuclear medicine study, diagnostic reference levels (DRLs) were computed. DRLs are based on actual-patient dose distributions from the data collected for specific procedures and set at the 75th percentile of these measured data, meaning that the procedures are performed at most institutions with doses at or below the reference levels (6-8). The purpose of the DRL is to provide a benchmark for comparison, not to define a maximum or minimum dose limit.

Unpaired, 2-tailed Student $t$ testing with unequal variance analysis was performed between the activity and dose distribution and between the 2010 and 2014 results for each surveyed study. A $P$ value lower than 0.05 , corresponding to a $95 \%$ confidence interval, was considered statistically significant.

\section{RESULTS}

\section{Participation}

Forty-six percent (22/48) of the surveyed centers completed the first form (about the administered dose in nuclear medicine studies), and of those, 19 centers also completed the second form (about the CT parameters used in selected SPECT/CT studies). This $46 \%$ response rate for the first form was lower than in the 2010 survey, in which $63 \%$ of the centers (30/48) completed the first form. However, the response rate for the second form was higher than in 2010, when SPECT/CT cameras were not as widely available. Only 12 of the 30 surveyed centers completed the second form in 2010, as compared with 19 of the 22 surveyed centers in 2014.

\section{Radiopharmaceutical Administered Dose Survey}

Table 1 gives the distribution (minimum, maximum, mean, and SD) of administered activity for the most common nuclear medicine studies and the most recent conversion factor, along with the distribution (mean and SD) of the corresponding effective dose. DRLs are given in terms of both activity and effective dose. These 2014 results are followed by the 2010 results, with both activity distribution and corresponding effective dose displayed. The rightmost column is the result of the Student $t$ test analysis between the dose distributions of 2010 and 2014 for each surveyed study.

Figure 1 shows the activity and dose distributions of the surveyed nuclear medicine studies. On the left is the administered activity distribution, with the $x$-axis oriented toward the left with increasing activity. On the right is the distribution of the corresponding effective dose, with the $x$-axis oriented toward the right with increasing dose. The grayed boxes represent the SD around the mean, and the whiskers give the minimum and maximum values from the survey responses.

Careful analysis of Table 1 reveals that 2 radiopharmaceuticals harbored a significantly higher conversion factor than the remainder: ${ }^{67} \mathrm{Ga}$-citrate and ${ }^{131} \mathrm{I}$. The conversion factor is significantly higher for an agent that decays with the emission of $\beta^{-}$particles, such as ${ }^{131} \mathrm{I}$. This factor varies with the level of thyroid function: $14 \mathrm{mSv} \mathrm{MBq}^{-1}$ for low thyroid function, $22 \mathrm{mSv} \mathrm{MBq}^{-1}$ for medium thyroid function, and $29 \mathrm{mSv} \mathrm{MBq}^{-1}$ for high thyroid function. Because most thyroid scans in nuclear medicine are performed either to characterize a thyroid nodule or to evaluate for hyperthyroidism, we chose to use the medium conversion factor in this comparative study. The other tracer that harbored a significantly higher conversion factor than most of the $\gamma$-emitters was ${ }^{67} \mathrm{Ga}$-citrate, with a factor of $0.100 \mathrm{mSv} \mathrm{MBq}^{-1}$, which is at least 10 times higher than the average of the other $\gamma$-emitter radiopharmaceuticals.

The remainder of the ${ }^{99 \mathrm{~m}}$ Tc-based radiopharmaceuticals delivered average effective doses clustered below $10 \mathrm{mSv}$ (vertical dotted line in Fig. 1). However, we should mention that there is a cumulative effect from the stress and the rest portions of myocardial perfusion studies.

Two studies - myocardial perfusion imaging and ${ }^{67} \mathrm{Ga}-$ citrate imaging - showed a statistically significant difference between 2010 and 2014, with a $P$ value lower than 0.05 .

Cardiac Imaging. Twenty-one centers used ${ }^{99 \mathrm{~m}}$ Tc-based radiopharmaceuticals, and only 1 center used ${ }^{201} \mathrm{Tl}$, which was excluded from this comparative analysis. Nineteen centers performed rest and stress studies in a 2-d protocol with a mean effective dose of $14.6 \pm 2.9 \mathrm{mSv}$, and 2 other centers used a 1-d protocol with a mean effective dose of $13.7 \pm 0.2 \mathrm{mSv}$. The distribution of effective dose did not significantly differ between the 1-d and 2-d protocols $(P=0.189)$.

With regard to methods of image acquisition and reconstruction, 11 centers used a regular $\gamma$-camera without resolution recovery reconstruction, 5 used a regular $\gamma$-camera with resolution recovery reconstruction, and 5 used the proprietary IQ SPECT camera (Siemens). At the time of our survey, no center was using the semiconductor camera from GE Healthcare. There was no statistically significant difference between use and nonuse of resolution recovery reconstruction $(P=0.171)$. The only statistically significant difference (Table 2) was between use of a $\gamma$-camera, regardless of resolution recovery reconstruction, and use of an IQ SPECT camera $(P=0.008)$.

Overall, combining both radiopharmaceuticals and both the stress and the rest studies, the average effective dose received in 2014 was $14.5 \pm 2.7 \mathrm{mSv}$, with a DRL of 15.6 $\mathrm{mSv}$. Compared with the 2010 survey, which indicated an average effective dose of $18.3 \pm 3.9 \mathrm{mSv}$ and a DRL of $20.0 \mathrm{mSv}$, this diminution of dose is statistically significant $(P=0.0002)$. 


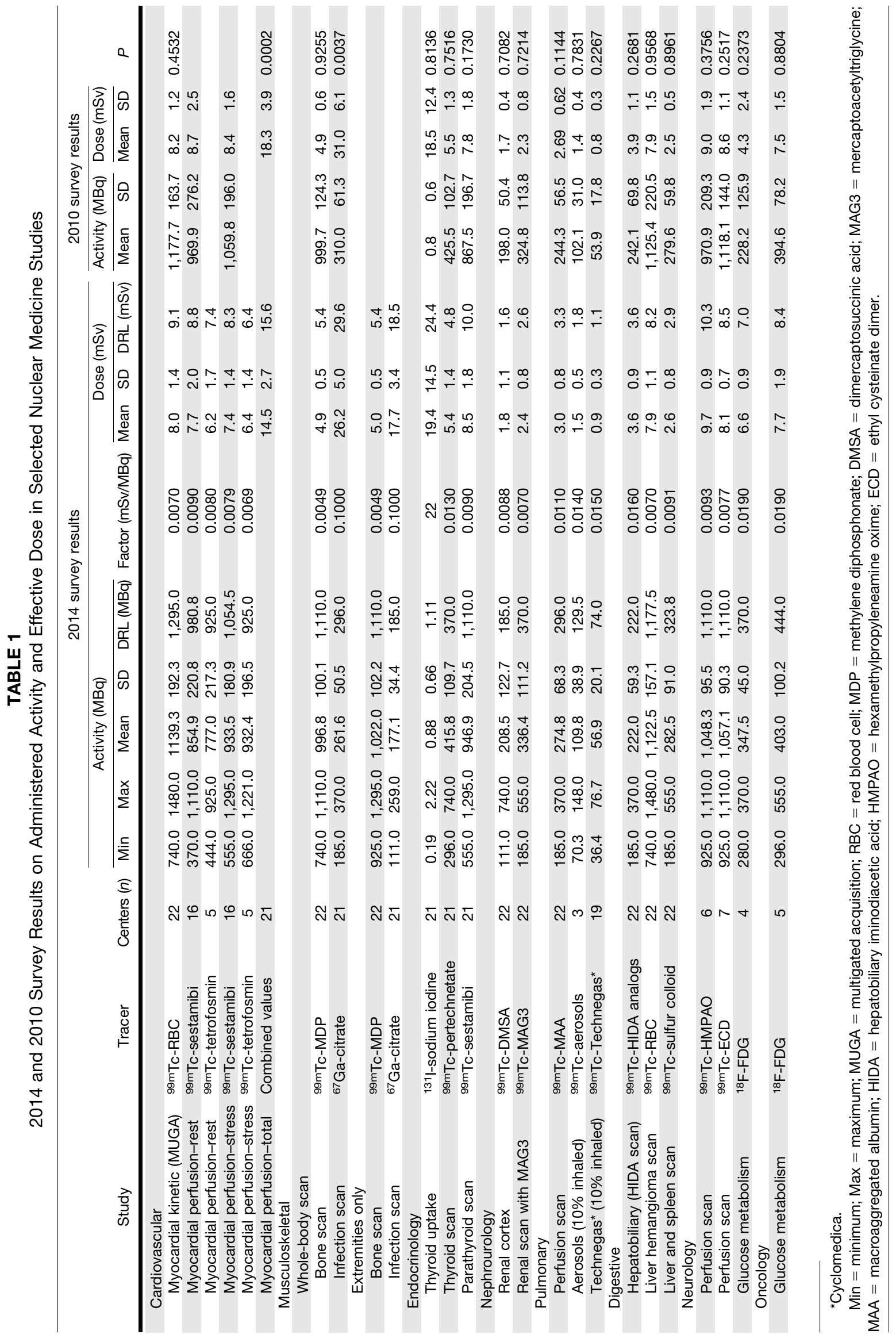




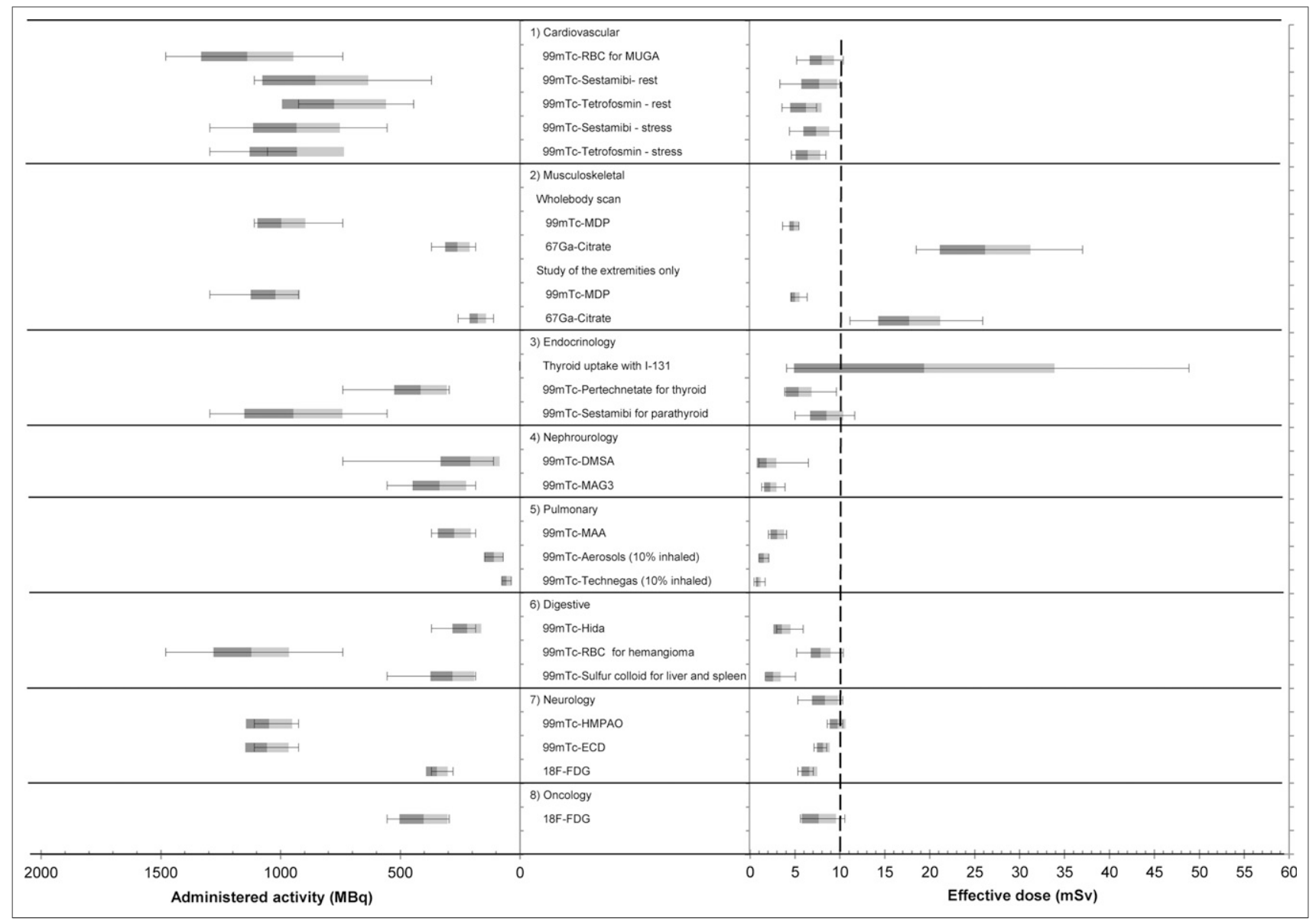

FIGURE 1. 2014 survey results: distribution of administered activity and effective doses in selected nuclear medicine studies. MUGA = multigated acquisition; DMSA = dimercaptosuccinic acid; MAG3 = mercaptoacetyltriglycine; MAA = macroaggregated albumin; RBC = red blood cells; HMPAO = hexamethylpropyleneamine oxime; ECD = ethyl cysteinate dimer.

Musculoskeletal and Infection Imaging. ${ }^{99 \mathrm{~m}} \mathrm{Tc}-\mathrm{methylene}$ diphosphonate was the agent used by all centers for bone scans. Nineteen centers performed bone scans with a fixed dose regardless of whether the imaging was performed for the whole body or for only the extremities, and 3 centers used a higher dose for the extremities. The whole-body dose reported by the 22 centers yielded a mean effective dose of $4.9 \pm 0.5 \mathrm{mSv}(996.8 \pm 100.1 \mathrm{MBq})$, with a DRL of 5.4 $\mathrm{mSv}$. This result is not significantly different from the 2010 result, which was a mean effective dose of $4.9 \pm 0.6 \mathrm{mSv}$ $(999.7 \pm 124.3 \mathrm{MBq})(P=0.9255)$.

TABLE 2

Effective Dose Difference Between Imaging Protocols for Combined Stress and Rest Myocardial Perfusion Studies

\begin{tabular}{ccc}
\hline \multicolumn{1}{c}{ Imaging protocol } & Centers $(n)$ & $\begin{array}{c}\text { Mean effective } \\
\text { dose }(\mathrm{mSv})\end{array}$ \\
\hline $\begin{array}{c}\mathrm{Nal}(\mathrm{TI}) \text { without resolution } \\
\text { recovery tool }\end{array}$ & 11 & $14.6 \pm 2.3$ \\
$\begin{array}{c}\mathrm{NaI}(\mathrm{TI}) \text { with resolution } \\
\text { recovery tool }\end{array}$ & 5 & $16.7 \pm 2.7$ \\
IQ SPECT & 5 & $12.1 \pm 1.6$ \\
\hline
\end{tabular}

For infection imaging, 21 centers used ${ }^{67} \mathrm{Ga}$-citrate and only 1 used labeled white blood cells. Of the 21 centers that used ${ }^{67} \mathrm{Ga}$-citrate, 16 applied a whole-body-imaging dose different from the extremity-imaging dose, and 5 applied the same dose for both.

Overall, the mean effective dose was $26.2 \pm 5.0 \mathrm{mSv}$ $(261.6 \pm 50.5 \mathrm{MBq})$ for whole-body imaging and $17.7 \pm$ $3.4 \mathrm{mSv}(177.1 \pm 34.4 \mathrm{MBq})$ for extremity imaging. This difference is significant $\left(P=2.9 \times 10^{-7}\right)$.

In 2010, the questionnaire had fewer directives than in 2014, and the information collected allowed us to compare only the whole-body protocol, which had a mean effective dose of $31.0 \pm 6.1 \mathrm{mSv}(310.0 \pm 61.3 \mathrm{MBq})$. The decrease in delivered dose since 2010 is significant $(P=0.0037)$.

\section{CT Portion of SPECT/CT Survey}

Among the 19 centers that completed our second form, the distribution of camera brands was as follows: 5 Infinia Hawkeye (GE Healthcare), 3 Discovery NM/CT 670 (GE Healthcare), 4 Symbia T (Siemens), 3 Symbia T2 (Siemens), 2 Symbia T6 (Siemens), 1 Precedence (Phillips), and 1 Brightview (Phillips). Data from the 2 Phillips cameras and from 1 Siemens Symbia T were too incomplete, and these 3 
centers were excluded from the analysis. The results from the remaining 16 centers were included in the analysis. Preliminary review of the responses showed a wide variability in the parameters used within the same imaging protocol. Reported CTDI and DLP were not representative of the actual protocol but rather of random patients' dose reports. It was thus decided to recalculate all CTDIs without including the effect of current modulation.

Current, Rotation Time, and Pitch. The choices for current settings differed across vendors, and the surveyed centers had difficulty retrieving the information with confidence. Current modulation, although not available on the Infinia Hawkeye, is also a factor to consider, as it alters the delivered current in a given patient. Seven of the 11 centers (the 16 surveyed centers minus the 5 with Infinia Hawkeyes) that had the modulation technology available answered that it was indeed used clinically. However, we know from previous visits to centers and discussions with application technicians that this number is underestimated, thus raising questions about the understanding of current modulation by on-site teams.

To assess the weight of current as a parameter in the overall assessment of effective dose, we transformed all responses into effective $\mathrm{mAs}$. Effective $\mathrm{mAs}$ is the product of the current in milliamps $(\mathrm{mA})$ multiplied by the rotation speed (rotations per second) and divided by the pitch (inward table-motion per rotation, divided by photon-beam thickness). The mean effective mAs was thus $31.6 \pm 18.8$, again without the effect of current modulation.

Tension. The tension setting was either 120 or $140 \mathrm{kVp}$ for GE Healthcare cameras and either 110 or $130 \mathrm{kVp}$ for Siemens cameras. The only exception was a single center that used $80 \mathrm{kVp}$ for parathyroid scintigraphy.

Collimation and Slice Thickness. Most centers used a collimation that allowed them to recover a slice thickness ranging from 2.5 to $10 \mathrm{~mm}$, with a median of $5 \mathrm{~mm}$.

Length of Scan. To double the length of the scan along the craniocaudal axis is to double the dose to the patient (forgoing the current modulation effect). This variable was difficult to assess in our survey because some centers used a protocol-based fixed scan length and others used a patientspecific scan length based on the planar imaging findings or the clinical question. To compare SPECT/CT protocols between these centers, we chose to define a fixed $20-\mathrm{cm}$ scan length for all studies except ${ }^{67} \mathrm{Ga}$-citrate studies, for which the scan length was set to $30 \mathrm{~cm}$ to reflect to most probable use of ${ }^{67} \mathrm{Ga}$-citrate SPECT/CT imaging: infection.

CTDI, DLP, and Effective Dose. The calculated CTDIs without current modulation were $3.9 \pm 2.0 \mathrm{mGy}$ for parathyroid SPECT/CT, $2.4 \pm 1.4 \mathrm{mGy}$ for attenuation correction in myocardial perfusion studies, and $2.7 \pm 1.2 \mathrm{mGy}$ for localization of ${ }^{67} \mathrm{Ga}$ uptake in the chest. The calculated DLPs were $78.5 \pm 40.6 \mathrm{mGy} \times \mathrm{cm}$ for parathyroid SPECT/CT, $49.6 \pm 28.1 \mathrm{mGy} \times \mathrm{cm}$ for attenuation correction in myocardial perfusion studies, and $83.4 \pm 37.2 \mathrm{mGy} \times \mathrm{cm}$ for localization of ${ }^{67} \mathrm{Ga}$ uptake in the chest. These values are meaningless because they do not account for differences in tissue sensitivity, as exemplified by a lower sensitivity in neck organs than in chest organs. The ImPACT CT dosimetry tool was thus used to determine the upper and lower acquisition limits usually used in SPECT/CT protocols, in order to convert the DLPs into useful effective doses (Table 3).

For parathyroid SPECT/CT, the CT portion yielded a mean effective dose of $0.6 \pm 0.4 \mathrm{mSv}$, which was 14 times lower than the mean effective dose from the radiopharmaceutical portion, $8.5 \pm 1.8 \mathrm{mSv}$. For attenuation correction of myocardial perfusion studies, the CT portion yielded a mean effective dose of $1.0 \pm 0.6 \mathrm{mSv}$, which was again 14 times lower than the mean effective dose from the radiopharmaceutical portion, $14.5 \pm 2.7 \mathrm{mSv}$. For localization of ${ }^{67} \mathrm{Ga}$ uptake in infectious studies of the chest, the CT portion yielded a mean effective dose of $1.8 \pm 1.2 \mathrm{mSv}$, which was 14.5 times lower than the mean effective dose from the radiopharmaceutical portion, $26.2 \pm 5.0 \mathrm{mSv}$.

\section{DISCUSSION}

Despite having a low mean administered activity, some studies yielded a correspondingly high effective dose. The physical characteristics of the radioisotope (half-life, type of radiation emission), as well as the characteristics of the given pharmaceutical (organ uptake distribution, elimination pathways, biologic half-life), explain the difference in dosimetry. The use of effective dose, obtained by multiplying the administered activity by the appropriate conversion factor, allows dosimetry comparisons between protocols that use different radiopharmaceuticals (9). A good example is ${ }^{131} \mathrm{I}$, which has the highest conversion factor given its radiation emission of $\beta$-particles in addition to the $\gamma$-rays. Despite the administration of a very small amount of activity,

TABLE 3

CTDI, DLP, and Effective Dose in Various CT Protocols Performed in SPECT/CT

\begin{tabular}{|c|c|c|c|c|c|c|c|}
\hline \multirow[b]{2}{*}{ Region } & \multirow[b]{2}{*}{ SPECT/CT protocol } & \multicolumn{2}{|c|}{ CTDI (mGy) } & \multicolumn{2}{|c|}{ DLP (mGy × cm) } & \multicolumn{2}{|c|}{$\begin{array}{l}\text { Effective dose } \\
\quad(\mathrm{mSv})\end{array}$} \\
\hline & & Average & SD & Average & SD & Average & SD \\
\hline Neck and thorax $(20 \mathrm{~cm})$ & Parathyroid study & 3.9 & 2.0 & 78.5 & 40.6 & 0.6 & 0.4 \\
\hline Thorax $(20 \mathrm{~cm})$ & $\begin{array}{l}\text { Attenuation correction for myocardial } \\
\text { study }\end{array}$ & 2.4 & 1.4 & 49.6 & 28.1 & 1.0 & 0.6 \\
\hline Thorax (30 cm) & ${ }^{67} \mathrm{Ga}$-citrate for infection study & 2.7 & 1.2 & 83.4 & 37.2 & 1.8 & 1.2 \\
\hline
\end{tabular}


${ }^{131}$ I yields an unfavorably high corresponding effective dose across a wide range of thyroid function. Another example, ${ }^{67} \mathrm{Ga}$-citrate, has a conversion factor of $0.100 \mathrm{mSv} \mathrm{MBq}^{-1}$, which is at least 10 times higher than the average of the other $\gamma$-emitter tracers.

Excluding ${ }^{131} \mathrm{I}$ for thyroid studies and ${ }^{67} \mathrm{Ga}$-citrate for both infectious workups, the remainder of the ${ }^{99 \mathrm{~m}} \mathrm{Tc}$-based nuclear medicine radiopharmaceuticals used routinely in the surveyed centers delivered effective doses clustered below $10 \mathrm{mSv}$. Again, we should mention that there is a cumulative effect from the stress and the rest portions of myocardial perfusion studies, with the 2 portions yielding a combined effective dose of $14.5 \pm 2.7 \mathrm{mSv}$.

The survey showed that between 2010 and 2014, there was a significant decrease in the delivered effective dose in myocardial perfusion studies and in ${ }^{67} \mathrm{Ga}$-citrate scans for infectious workup. For myocardial perfusion studies, we hypothesize that the decrease was partly related to the increase in awareness brought about by our initial survey. In 2010 , the results that we presented at provincial and national meetings stressed the relatively high cumulative dose delivered by the combined stress and rest portions of myocardial perfusion studies. Another factor that could explain the decrease was the introduction of new imaging modalities from the camera manufacturers and the availability of resolution recovery software. The arrival of a new technology at a given center usually provides an occasion to review the imaging protocols. When the choice was between decreasing either the acquisition time or the injected activity, most centers chose to instead decrease both, with the resulting test being easier to bear by the patient and having an overall lower effective dose. A proposed alternative (10) is to perform a stress-only acquisition protocol in which a patient with a normal stress result can forgo the second, rest, portion of the test. This alternative has the potential to significantly lower the delivered effective dose.

Our report on the 2010 survey emphasized the high effective dose delivered in ${ }^{67} \mathrm{Ga}$-citrate studies. Current reconstruction software allows resolution recovery of images with a lower count rate $(11-13)$. There is, however, a limit to which the injected dose of a given tracer can be lowered without impeding the diagnostic accuracy of the imaging study. This limit was probably reached by the centers that had commendably decreased their injected activity of ${ }^{67} \mathrm{Ga}$-citrate. There is probably still room for improvement in many centers, although individual protocols are to be modulated according to the available camera technology and particular population specifics. Labeled white blood cells are an alternative $(14,15)$ but are less easily available given the time-consuming labeling process and the significantly higher associated cost. ${ }^{18} \mathrm{~F}-\mathrm{FDG}$ $\mathrm{PET} / \mathrm{CT}$ is also an alternative $(16,17)$ but is even less widely available. We certainly will encourage our colleagues to continue the optimization of infectious protocol imaging and dosimetry.

Most of the other nuclear medicine tests did not show significant changes in dosimetry between 2010 and 2014 . This finding is surprising, as the same resolution recovery tools as are available for ${ }^{67} \mathrm{Ga}$-citrate studies are also applicable to most $\gamma$-emitter imaging protocols. These protocols are, however, in the lower dose range and were probably not selected initially for optimization.

The CT portion of SPECT/CT protocols contributes a relatively small fraction of the total delivered effective dose of a given nuclear medicine study $(18,19)$. However, there was wide variation in the parameters used across the centers surveyed. Optimization of the delivered dose from CT is again certainly possible and will likely require further education about the effect of the different parameters that underlie the choice of image quality and corresponding dose in CT.

Protocol optimization is always based on a typical patient encountered for a given medical indication. Diagnostic accuracy in a particular patient should take precedence over dosimetry concerns, when applicable. Conversion factors remain a crude way to assess the corresponding effective dose in a healthy adult population, as the dose distribution in a given patient is relative to the physiologic function of many organs, especially the liver and the kidneys, which are responsible for elimination of most of the radiopharmaceutical.

CTDI, DLP, and corresponding effective dose in CT are also estimates, as these values are derived from averaged measurements at specific CT parameters in acrylic-glass phantoms. Thus, patient protocol reports of CTDI and DLP state only what would have been the delivered dose to a phantom at the chosen acquisition parameters. However, the aim of this survey was not to perform accurate individual dosimetry but to compare protocols between different radiopharmaceuticals and between different nuclear medicine and CT modalities. For this aim, the only acceptable choice is to compute the corresponding effective doses, keeping in mind the above-discussed limitations.

\section{CONCLUSION}

We now have a more recent understanding of the distribution of mean effective doses in various SPECT and SPECT/CT studies across the province. Between our 2010 and 2014 surveys, there was a significant decrease in the delivered effective dose in myocardial perfusion studies and in ${ }^{67} \mathrm{Ga}-$ citrate scans for infectious workup. The CT portion of SPECT/ CT protocols contributed a relatively small fraction of the total delivered effective dose of a given nuclear medicine study.

We hypothesize that technologic advances combined with education and collegial discussions between technologists and nuclear medicine physicians will allow our specialty to meet the challenge of dosimetry optimization. As the technology continuously evolves, the nuclear medicine community faces continuous challenges regarding the acquisition and reconstructions parameters of our studies. Technologic evolution provides us with the opportunity to improve the quality and accuracy of our diagnostic tests, as well as the occasion to review the associated dosimetry. There is certainly room for further improvement, and we will continue to promote the safest use of nuclear medicine studies at our centers. 


\section{DISCLOSURE}

No potential conflict of interest relevant to this article was reported.

\section{ACKNOWLEDGMENT}

We thank the surveyed centers for their participation.

\section{REFERENCES}

1. Mattsson S, Johansson L, Leide Svegborn S, et al. Radiation dose to patients from radiopharmaceuticals: a compendium of current information related to frequently used substances-ICRP publication 128. Ann ICRP. 2015;44:7-321.

2. Radiation dose to patients from radiopharmaceuticals: ICRP publication 53. Ann ICRP. 1987;18:1-377.

3. Radiation dose to patients from radiopharmaceuticals: addendum 2 to ICRP publication 53-ICRP publication 80. Ann ICRP. 1998;28:1-126.

4. Radiation dose to patients from radiopharmaceuticals: addendum 3 to ICRP publication 53-ICRP publication 106. Ann ICRP. 2008;38:1-197.

5. ImPACT CTDosimetry tool, version 1.0.4. Impactscan.org website. http://www. impactscan.org/ctdosimetry.htm\#CTDoseDownload. Updated May 27, 2011. Accessed February 12, 2018

6. Roch P, Aubert B. French diagnostic reference levels in diagnostic radiology, computed tomography and nuclear medicine: 2004-2008 review. Radiat Prot Dosimetry. 2013;154:52-75.

7. Morin RL, Seibert JA, Boone JM. Radiation dose and safety: informatics standards and tools. J Am Coll Radiol. 2014;11:1286-1297.

8. Goske MJ, Strauss KJ, Coombs LP, et al. Diagnostic reference ranges for pediatric abdominal CT. Radiology. 2013;268:208-218.
9. McCollough CH, Schueler BA. Calculation of effective dose. Med Phys. 2000; 27:828-837.

10. Dorbala S, Blankstein R, Skali $\mathrm{H}$, et al. Approaches to reducing radiation dose from radionuclide myocardial perfusion imaging. J Nucl Med. 2015;56: 592-599.

11. Armstrong IS, Saint KJ, Tonge CM, Arumugam P. Evaluation of general-purpose collimators against high-resolution collimators with resolution recovery with a view to reducing radiation dose in myocardial perfusion SPECT: a preliminary phantom study. J Nucl Cardiol. 2017;24:596-604.

12. Pagnanelli R, Borges-Neto $\mathrm{S}$. Technical aspects of resolution recovery reconstruction. J Nucl Cardiol. 2016;23:149-152.

13. Zoccarato O, Scabbio C, De Ponti E, et al. Comparative analysis of iterative reconstruction algorithms with resolution recovery for cardiac SPECT studies: a multi-center phantom study. J Nucl Cardiol. 2014;21:135-148.

14. Bar-Shalom R, Yefremov N, Guralnik L, et al. SPECT/CT using ${ }^{67} \mathrm{Ga}$ and ${ }^{111} \mathrm{In}-$ labeled leukocyte scintigraphy for diagnosis of infection. J Nucl Med. 2006;47: 587-594.

15. Palestro CJ. Radionuclide imaging of musculoskeletal infection: a review. $J$ Nucl Med. 2016;57:1406-1412.

16. Vaidyanathan S, Patel CN, Scarsbrook AF, Chowdhury FU. FDG PET/CT in infection and inflammation: current and emerging clinical applications. Clin Radiol. 2015;70:787-800.

17. Vos FJ, Bleeker-Rovers CP, Corstens FHM, Kullberg BJ, Oyen WJG. FDG-PET for imaging of non-osseous infection and inflammation. $Q \mathrm{~J} \mathrm{Nucl} \mathrm{Med} \mathrm{Mol}$ Imaging. 2006;50:121-130.

18. Rausch I, Fuchsel FG, Kuderer C, Hentschel M, Beyer T. Radiation exposure levels of routine SPECT/CT imaging protocols. Eur J Radiol. 2016;85:16271636 .

19. Montes C, Tamayo P, Hernandez J, et al. Estimation of the total effective dose from low-dose CT scans and radiopharmaceutical administrations delivered to patients undergoing SPECT/CT explorations. Ann Nucl Med. 2013;27: $610-617$. 